\title{
A Novel Noise Removal Method Using Neural Networks
}

\author{
Catalina COCIANU, Alexandru STAN \\ Bucharest University of Economic Studies \\ catalina.cocianu@ie.ase.ro, alexandru.stan1@yahoo.com
}

In this paper is presented a new technique consisting in applying some pre-whitening and shrinkage methods followed by a neural network-based supervised approach for correlated noise removal purposed. In our work the type of noise and the covariance matrix of noise are known or can be estimated using the "white wall" method. Due to data dimensionality, a PCAbased compression technique is used to obtain a tractable solution. The local memories of the neurons are determined using a supervised learning process based on the compressed preprocessed inputs and the compressed version of the original images. The proposed method is evaluated using some of the most commonly used indicators and the results are reported in the third section of the paper. The conclusive remarks together with suggestions for further work are supplied in the final part of the paper.

Keywords: Digital Image Processing, Correlated Noise Removal, Supervised Learning, Neural Networks, Shrinkage Techniques

1 Introduction

Different types of imaging systems, as for instance digital cameras, medical imaging systems, infrared and radar remote sensing complexes induce noise in resulted digital images. Moreover, the acquisition and codification of images in digital formats and the transmission of the obtained images files through some communication channels usually affected by noise are also known as processes that degrade images usually by additive noise. Because of this, the noise removal procedure is one of the most commonly used pre-processing step in solving digital image processing tasks. Therefore, a significant part of digital image procedures are dedicated to image denoising, most of them being developed under the assumptions that the images are corrupted by additive white noise [1], [2], [3], [4]. In our work, we keep the assumption about normality, but we consider that the superimposed noise affects neighbour image pixels in a correlated manner.

During the past decade there have been reported a series of correlated noise removal methods, most of them being based on timefrequency and spatial-frequency image features respectively. One of the first waveletbased techniques for correlated noise removal purposes was developed using a pre-whiten- ing procedure followed by the wavelet thresholding [5]. Another method models the noisefree coefficients using a multivariate Gaussian Scale Mixture [6]. Also, image denoising using Hidden Markov Models in the wavelet domain based on the concept of signal of interest were reported in [7]. Other class of methods uses Discrete Cosine Transform (DCT) for noise removal in case the spatial correlation characteristics of the noise component can be estimated [8], [9]. More recently, a series of works concerning the sparsity of signals exploited for noise removal purposes based on spatial-frequency image features and princi$\mathrm{pal} /$ independent components analysis were reported in [10], [11]. Higher criticism method for detecting signals that are both sparse and weak has been proposed to identify sparse signals in correlated noise, in more general denoising depending data problem context [12],[13]. Also, machine learning techniques such as neural architectures, support vector machines (SVM) and more sophisticated models were developed for solving different image processing tasks, including image restoration [14], [15], [16].

The aim of the paper is to report a pre-whitening procedure based on simultaneous diagonalization technique combined with a supervised learning method using neural networks for spatially correlated noise removal. In our 
approach the type of noise and the covariance matrix of noise are known or can be estimated using the "white wall" method. Due to data dimensionality, a Principal Component Analysis (PCA)-based compression technique is used to obtain a tractable solution [17].

\section{The Proposed Methodology for Corre- lated Noise Removal Purposes}

In our approach, the observed noisy images are considered to be grouped in $d$ classes, each class being modelled as a $n$-dimensional random vector and the entire set of images is represented in terms of a certain mixture vector

$$
\mathrm{x}=\mathrm{x}_{0}+\eta(1)
$$

where $\mathrm{x}_{0}$ models the set of the original cleaned images and $\eta$ is the Gaussian noise component, $\eta \sim N\left(0, \Sigma_{\eta}\right)$. Note that we consider the linear representation of digital images when defining the model (1). The working assumptions concerning the model (1) are that $x_{0}, \eta$ are independent and the noise covariance matrix $\Sigma_{\eta}$ can be estimated. One of the most commonly used technique to estimate the noise covariance matrix is the so-called "white wall technique". If we consider $\mu_{0}=$ $E\left(\mathrm{x}_{0}\right), \Sigma_{0}=E\left(\left(\mathrm{x}_{0}-\mu_{0}\right) \cdot\left(\mathrm{x}_{0}-\mu_{0}\right)^{T}\right), \mu=$ $E(\mathrm{x})$, and $\Sigma=E\left((\mathrm{x}-\mu) \cdot(\mathrm{x}-\mu)^{T}\right)$, the parameters $\mu$ and $\Sigma$ can be estimated from available data and $\mu_{0}, \Sigma_{0}$ are estimated based on $\Sigma_{\eta}$,

$$
\mu_{0} \cong \mu, \Sigma_{0} \cong \Sigma-\Sigma_{\eta}
$$

Let us denote by $\mathrm{S}$ the set of observed images. The basic working assumptions are that $\mathrm{S}$ contains a subset $S_{1}$ of observed images whose original, cleaned versions are available, and at least one image of each class belongs to $S_{1}$. We denote by $S_{0}$ the available original images and let $S_{2}=S \backslash S_{1}$. The prosed methodology is of supervised type and consists in the following stages.

Stage I. The training stage:

1. Apply a certain correlated noise reduc- tion procedure, CNRP, to the images belonging to $S_{1}$ and get the set of pre-processed images $C S_{1}$.

2. Train a feed forward neural network, to learn the associations $(I, J)$, where $I \in$ $C S_{1}, J \in S_{0}$ and they correspond to a certain noisy image in $S_{1}$, that is there exists $I_{\text {noisy }} \in S_{1}$ such that $J$ is the cleaned version of $N I$ and $I=C N R P\left(I_{\text {noisy }}\right)$. Let $f_{N N}$ be

the resulted function.

Stage II. The testing stage, where new inputs are considered

For each $I \in S_{2}$

3. Compute $I_{1}=C N R P(I)$

4. Compute an approximation of the cleaned version of $I_{1}, I_{\text {cleaned }}=f_{N N}\left(I_{1}\right)$ Basically, the proposed methodology consists in a pre-processing step, where the noise component is partially eliminated, followed by a neural network-based testing/forecasting procedure, applied in order to estimate and remove the still remaining noise.

One of the main issues when digital images are used in neural network-based learning procedures is the data dimensionality. In order to reduce the computational complexity, a PCAbased compression/decompression technique is applied.

\subsection{Simultaneous Diagonalization-Based Technique and Code Shrinkage Method for Correlated Noise Reduction}

The pre-processing step implements a decorrelation-based noise reduction technique [18]. In case of the degradation model (1), the correlated noise reduction procedure (CNRP) is described as follows.

Step 1. Data alignment and centring.

Compute

Obviously, we get

$$
\mathrm{Y}=\mathrm{x}-\mathrm{E}(\mathrm{x})
$$

$$
Y=x_{0}-\mu_{0}+\eta-\mu_{\eta}
$$

Since the noise component and the observed signal are independent, the covariance matrix of the random vector $\mathrm{Y}$ is computed by 


$$
\begin{gathered}
\operatorname{Cov}\left(\mathrm{Y}, Y^{T}\right)=\operatorname{Cov}\left(x_{0}-\mu_{0},\left(x_{0}-\mu_{0}\right)^{T}\right)+\operatorname{Cov}\left(\eta-\mu_{\eta},\left(\eta-\mu_{\eta}\right)^{T}\right) \\
\operatorname{Cov}\left(\mathrm{Y}, Y^{T}\right)=\Sigma_{0}+\Sigma_{\eta}
\end{gathered}
$$

Step2. The decorrelation process

Let $\mathrm{A}$ be the matrix simultaneously diagonalizing $\Sigma_{0}$ and $\Sigma_{\eta}$. Note that the columns of A are a set of eigenvectors of the matrix $\Sigma_{0}^{-1}$. $\Sigma_{\eta}$. We get

$$
\left\{\begin{array}{l}
A^{T} \Sigma_{0} A=I_{n} \\
A^{T} \Sigma_{\eta} A=\Lambda
\end{array}\right.
$$

where $\Lambda=\operatorname{diag}\left\{\lambda_{1}, \lambda_{2}, \ldots, \lambda_{n}\right\}$ is the eigenvalues matrix of $\Sigma_{0}^{-1} \cdot \Sigma_{\eta}$.

We consider the transform

$$
Z=A^{T} \cdot Y
$$

Obviously,

$$
Z=A^{T}\left(x_{0}-\mu_{0}\right)+A^{T}\left(\eta-\mu_{\eta}\right)
$$

where $A^{T}\left(x_{0}-\mu_{0}\right), A^{T}\left(\eta-\mu_{\eta}\right)$ are independent and the noise component is mainly contained by the term $A^{T}\left(\eta-\mu_{\eta}\right)$ in (8).

The covariance matrix $\mathrm{Z}$ is

$$
\operatorname{Cov}\left(\mathrm{Z}, Z^{T}\right)=A^{T} \Sigma_{0} A+A^{T} \Sigma_{\eta} A
$$

Using (5) and (6), we get

$$
\operatorname{Cov}\left(\mathrm{Z}, Z^{T}\right)=I_{n}+\Lambda
$$

Let us denote by $\eta^{\prime}$ the noise term,

$$
\eta^{\prime}=A^{T}\left(\eta-\mu_{\eta}\right)=A^{T} \eta
$$

The covariance matrix of $\eta^{\prime}$ is

$$
\operatorname{Cov}\left(\eta^{\prime}, \eta^{\prime T}\right)=A^{T} \operatorname{Cov}\left(\eta-\mu_{\eta},\left(\eta-\mu_{\eta}\right)^{T}\right) A=A^{T} \Sigma_{\eta} A=\Lambda
$$

Since A is a constant matrix, $\eta \sim N\left(0, \Sigma_{\eta}\right)$ and using (10), we obtain that $\eta^{\prime} \sim \mathrm{N}(0, \Lambda)$. In other words, the additive noise component contained by the random vector $Z$ is basically white noise.

\section{Step 3. Code shrinkage}

The aim of the procedure is to remove the noise component from $\mathrm{Z}$ using a code shrinkage function defined as follows

$$
G(Z)=\left(g_{1}\left(z_{1}\right), \ldots, g_{n}\left(z_{n}\right)\right)^{T}
$$

where $Z=\left(z_{1}, z_{2}, \ldots, z_{n}\right)^{T}$ and $g_{i}\left(z_{i}\right)=$ $\operatorname{sign}\left(z_{i}\right) \max \left(0,\left|z_{i}\right|-\sqrt{2} \lambda_{i}\right), 1 \leq i \leq n$

Compute

$$
Z_{0}=G(Z)
$$

\section{Step 4. Noise reduction}

The obtained random vector $Y_{0}$ is computed based on the following relation

$$
Z_{0}=A^{T} Y_{0}
$$

Using (5) and (6) we get

$$
\begin{gathered}
A A^{T}=\Sigma_{0}^{-1} \\
A Z_{0}=A A^{T} Y_{0}=\Sigma_{0}^{-1} Y_{0}
\end{gathered}
$$

and, consequently,

$$
Y_{0}=\Sigma_{0} A Z_{0}
$$

Step 5. Image restauration
The restored version of $x_{0}$ is obtained by applying the inverse transform as follows.

Using (12) we get

$$
\hat{x}_{0}=Y_{0}+E(x)
$$

$$
\hat{x}_{0}=\Sigma_{0} A Z_{0}+E(x)
$$

From the implementation point of view, the above-mentioned method is applied as follows.

Let $O S=\left\{\left(x_{1}^{i}, x_{2}^{i}, \ldots, x_{N_{i}}^{i}\right), 1 \leq i \leq m\right\}$ be a set of noisy observed images modelled by a certain random vector $x$ defined by (1). For each $1 \leq i \leq m,\left(x_{1}^{i}, x_{2}^{i}, \ldots, x_{N_{i}}^{i}\right)$ represent the noisy versions of a certain image $x^{i}$ transmitted through a communication channel $N_{i}$ times, where $N_{i} \geq 1$. In case a new noisy image $x_{n e w}$ is received, the noise reduction procedure is applied based on the following scheme.

Stage 1. Apply CNRP to $\left(x_{1}^{i}, x_{2}^{i}, \ldots, x_{N_{i}}^{i}\right), 1 \leq$ $i \leq m$ and get $\left(\hat{x}_{1}^{i}, \hat{x}_{2}^{i}, \ldots, \hat{x}_{N_{i}}^{i}\right), 1 \leq i \leq m$. The corresponding CNRP procedure is characterized by the parameter $\mathrm{A}, \Lambda, \mathrm{G}, \mathrm{E}(\mathrm{x})$ and $\Sigma_{0}$

Stage 2. Apply CNRP to $x_{\text {new }}$ using the parameters computed at Stage 1 ,

$$
Y_{\text {new }}=x_{\text {new }}-E(x) Z_{\text {new }}=A^{T} Y_{\text {new }}
$$




$$
\begin{gathered}
Z_{0, \text { new }}=G\left(Z_{\text {new }}\right) \\
\hat{x}_{\text {new }}=\Sigma_{0} A Z_{0, \text { new }}+E(x)
\end{gathered}
$$

\subsection{The Neural Network-Based} Methodology for Noise Removal Purposes

A noise removal technique based on a neural architecture is introduced in this sub-section.

Artificial neural networks represent a machine learning technology with applications in such various areas, as for instance digital signal processing, time series analysis, pattern recognition. The main property of neural networks is the ability to learn from data with (supervised learning) or without (unsupervised learning) a teacher. The structure of a neural network is closely related to the learning algorithm used to train the network. From the structure point of view, there are three classes of neural architectures, namely single-layer feed forward networks, multilayer feed forward networks and recurrent networks. The single-layer feed forward architecture contains an input layer of nodes that projects onto an output layer containing computation nodes, being of acyclic type. The multilayer feed for- ward networks are characterised by the presence of at least one hidden layer whose computation nodes are hidden neurons. The hidden neurons are meant to somehow usefully intervene between the input external nodes and the output nodes. More precisely, the hidden computation nodes extract progressively more meaningful features from the input vectors. Recurrent networks differ from feed forward neural networks in that they have one or more feedback loops [19].

In our work we used a standard multilayer feed forward neural network having a single hidden layer, $F_{X} \rightarrow F_{H} \rightarrow F_{Y}$. The information moves from the nodes belonging to the input layer $F_{X}$ through the hidden layer $F_{H}$ and then to the output layer $F_{Y}$, without any feedback loops or cycles.

Let $\left|F_{X}\right|,\left|F_{Y}\right|$ and $\left|F_{H}\right|$ be the sizes of the input layer, output layer and hidden layer respectively. The number of neurons in the input layer corresponds to the input size, while the size of the output layer is given by the output dimension. The number of neurons in the hidden layer is usually set according to the following equations [20]

$$
\begin{array}{r}
\left|F_{H}\right|=2 \sqrt{\left(\left|F_{Y}\right|+2\right)\left|F_{X}\right|} \quad \text { (14) } \\
\left|F_{H}\right|=\sqrt{\left(\left|F_{Y}\right|+2\right)\left|F_{X}\right|}+2 \sqrt{\frac{\left|F_{X}\right|}{\left(\left|F_{Y}\right|+2\right)}} \\
\left|F_{H}\right|=\sqrt{\left|F_{X}\right| \cdot\left|F_{Y}\right|} \\
\left|F_{H}\right|=\frac{4 \cdot\left|F_{X}\right|^{2}+3}{\left|F_{X}\right|^{2}-8}, \text { when }\left|F_{Y}\right|=1
\end{array}
$$

Since in our approach $\left|F_{Y}\right|>1$, we consider a modified variant of (15) to establish the number of neurons in the hidden layer.

The supervised technique implemented to train the neural network is the LevenbergMarquard variant of the backpropagation algorithm. The Levenberg-Marquard algorithm is one of the most efficient optimization methods and provides a numerical solution of nonlinear least squares minimization problem based on Newton optimization technique. The iterative process is inspired by the hill-climbing procedure and uses Singular Value Decomposition technique to compute the updated weights of each connection. Let $S O=\left\{\left(x_{1}^{i}, x_{2}^{i}, \ldots, x_{N_{i}}^{i}\right), 1 \leq i \leq m\right\}$ be the set of noisy images. We denote by $\left\{x_{1}, \ldots, x_{m}\right\}$ the set of the original, cleaned images and let $\left(\hat{x}_{1}^{i}, \hat{x}_{2}^{i}, \ldots, \hat{x}_{N_{i}}^{i}\right), 1 \leq i \leq m$ be the pre-processed variants of images belonging to OS. The proposed neural network is designed to associate each image $\hat{x}_{j}^{i}, 1 \leq i \leq m, 1 \leq$ $j \leq N_{i}$ to its original variant, $x_{i}$, e.g. to compute $f_{N N}$ such that 


$$
x_{i}=f_{N N}\left(\hat{x}_{j}^{i}\right), 1 \leq i \leq m, 1 \leq j \leq N_{i}
$$

In other words, the neural architecture "learns" the difference between the images restored using the CNRP procedure and the original images.

A new image $x_{\text {new }}$ is cleaned using the proposed neural architecture by computing the resulted output of the network based on (18),

where

$$
x_{\text {cleaned }}=f_{\text {nN }}\left(\hat{x}_{\text {new }}\right)
$$

$$
\hat{x}_{\text {new }}=\operatorname{CNRP}\left(x_{\text {new }}\right)
$$

In order to reduce the computational complexity of the proposed neural network-based methodology, the images are compressed using a standard PCA method, the outputs being decompressed using the same PCA parameters. The procedure is described in [18].

\section{Experimentally Derived Results Con-} cerning the Performance of the Proposed Methodology

A long series of tests concerning the accuracy and generalization capabilities of the proposed neural network-based correlated noise removal procedure have been conducted. Some of the obtained results are presented in the following.
The tests were performed on a standard database, namely Senthil face images dataset, containing images of 5 human faces, 16 images for each person [21]. In the first test, the noise covariance matrix is assumed to be of $15 \times 10$ dimensions. In other words, the noise is assumed to be spatially correlated in $15 \times 10$ windowed neighbourhoods. The second experiment was conducted where $22 \times 20$ covariance matrix of the noise component is considered. The pre-processing step used 75 images of 5 persons, for each human face being used 15 of its available versions. The tests performed in order to evaluate the quality of the trained family of neural networks used the rest of 5 images, one for each person.

In our tests the working assumptions are that the noise affects the images at $15 \times 10$ and $22 \times 20$ block level respectively. Consequently, the pre-processing step and the neural network learning and testing procedures are implemented by splitting the images into $15 \times 10$ and $22 \times 20$ blocks.

The quality of a certain test image $T=$ $(t(x, y))$ computed in case $R=(r(x, y))$ is the reference image of the same size $\left(n_{x}, n_{y}\right)$ can be evaluated many ways. In our tests two of the most commonly used measures, namely Signal-to-Noise Ratio (SNR), and Root Mean Squared Signal-to-Noise Ratio (SNR_RMS) indicators were applied, where [1], [2]

$$
\begin{gathered}
\operatorname{SNR}(R, T)=10 * \log _{10}\left[\frac{\sum_{x=1}^{n_{x}} \sum_{y=1}^{n_{y}}(r(x, y))^{2}}{\sum_{x=1}^{n_{x}} \sum_{y=1}^{n_{y}}(r(x, y)-t(x, y))^{2}}\right] \\
S N R R \_R M S_{-}(R, T)=\sqrt{\frac{\sum_{x=1}^{n_{x}} \sum_{y=1}^{n_{y}}(r(x, y))^{2}}{\sum_{x=1}^{n_{x}} \sum_{y=1}^{n_{y}}(r(x, y)-t(x, y))^{2}}}
\end{gathered}
$$

In order to ensure a certain accuracy degree, the PCA compression thresholds $\varepsilon_{1}, \varepsilon_{2}$ are established such that $\varepsilon_{1} \cong 10^{-4}, \varepsilon_{2} \cong 10^{-5}$. The first threshold value corresponds to the pre-processed input images, while the second one is related to the original images, the outputs of the neural network. The compression thresholds are used to determine the number of neurons in the input and output layer respectively. The size of each hidden layer is established based on the following relation 


$$
\left|F_{H}\right|=\left[\frac{\sqrt{\left(\left|F_{Y}\right|+2\right)\left|F_{X}\right|}+2 \sqrt{\frac{\left|F_{X}\right|}{\left(\left|F_{Y}\right|+2\right)}}}{c}\right]
$$

where $c \cong 1.5$, due to the problem complexity.
Test 1. The noise component affects $15 \times 10$ windowed neighbourhoods

A noisy image belonging to SO, together with its pre-processed variant and the resulted cleaned version are presented in Figure1. In this case, the output image computed by the proposed neural architecture coincides with the original one, except the decompression error.
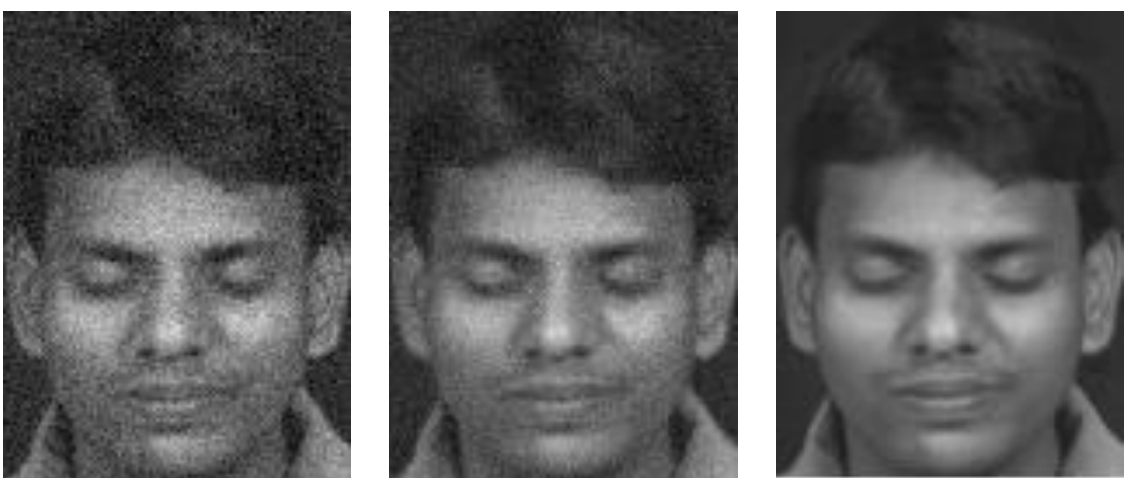

Fig. 1. The noisy image, the pre-processed image and the cleaned image

Three of the test images from the Senthil database and their versions resulted by applying the pre-processing step are shown in Figure 2 and Figure 3 respectively. Their cleaned versions computed by the resulted family of trained neural networks are shown in Figure 4. Note that the images are new, unseen yet, from the point of view of pre-processing step and from the neural network testing stage point of view. In other words, their corresponding original images are not known, some variants of them being computed using our proposed methodology. The quality of the restoration procedures expressed in terms of the indicators SNR and SNR RMS are summarized in Table 1.
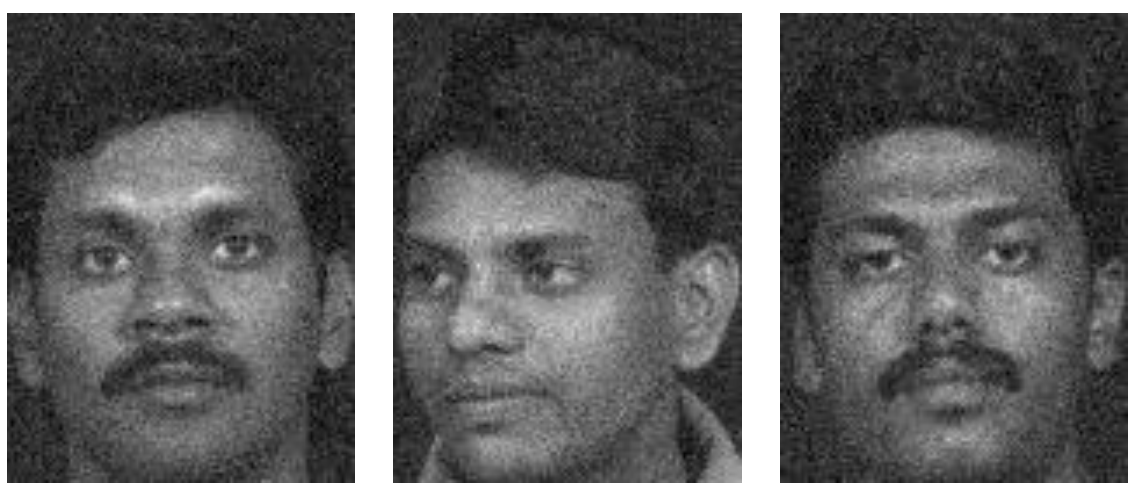

Fig. 2. New, unseen yet, noisy images 

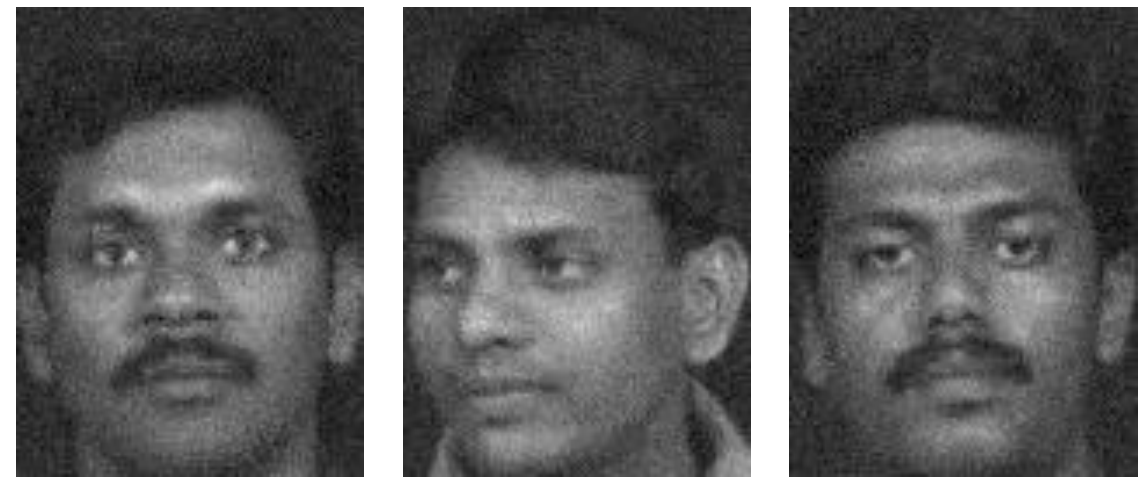

Fig. 3. The pre-processed variants of images shown in Figure 2
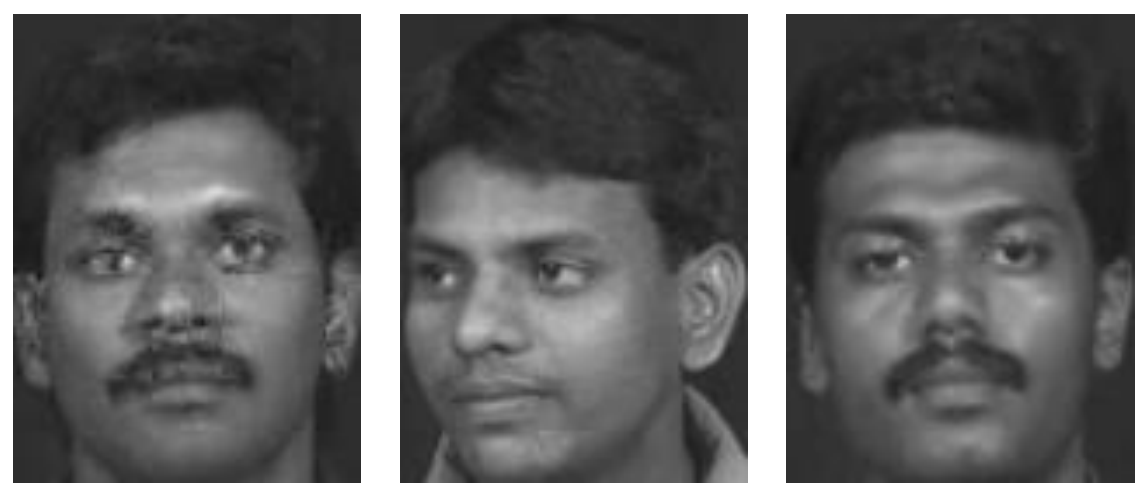

Fig. 4. The resulted cleaned images

Table 1. The qualitative analysis

\begin{tabular}{|l|c|c|}
\hline & SNR-RMS / image & SNR /image \\
\hline $\begin{array}{c}\text { Noisy images ver- } \\
\text { sus original images }\end{array}$ & $7.13,7.79,6.74$ & $17.06,17.83,16.58$ \\
\hline $\begin{array}{l}\text { Cleaned images (us- } \\
\text { ing the pre-processing } \\
\text { step) versus original } \\
\text { images }\end{array}$ & $8.40,9.64,8.30$ & $18.49,19.68,18.38$ \\
\hline $\begin{array}{l}\text { Cleaned images (us- } \\
\text { ing NN's) versus origi- } \\
\text { nal images }\end{array}$ & $12.46,33.71,28.34$ & $21.91,30.55,29.05$ \\
\hline
\end{tabular}

Test 2. The noise component affects $22 \times 20$ windowed neighbourhoods

In Figure 5 a noisy image belonging to SO, its pre-processed variant and the resulted cleaned version are depicted. Note that the output image computed by the proposed neural architecture coincides with the original one, except the decompression error.
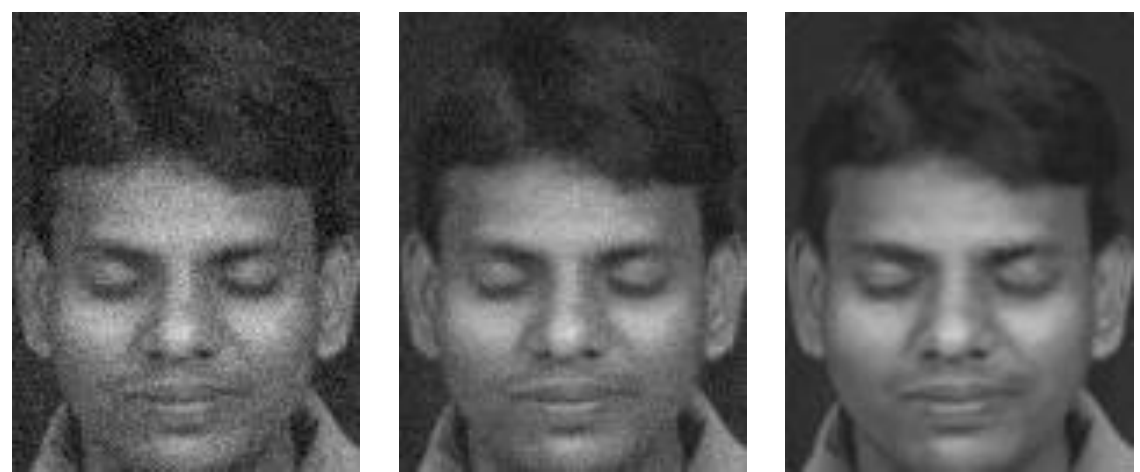

Fig. 5. The noisy image, the pre-processed image and the cleaned image 
Three of the test images from the Senthil database and their versions resulted by applying the pre-processing step are displayed in Figure 6 and Figure 7 respectively. Their versions computed by our method are shown in Figure
8. The corresponding original images of the signals presented in Figure 6 are not known. The quality of the restoration procedures expressed in terms of the indicators SNR and SNR_RMS are summarized in Table 2.
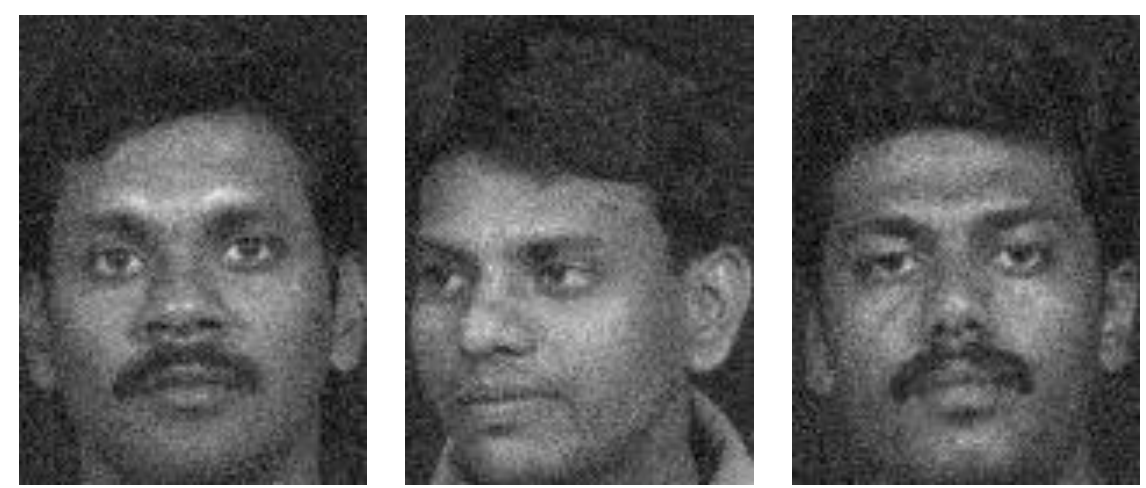

Fig. 6. New, unseen yet, noisy images
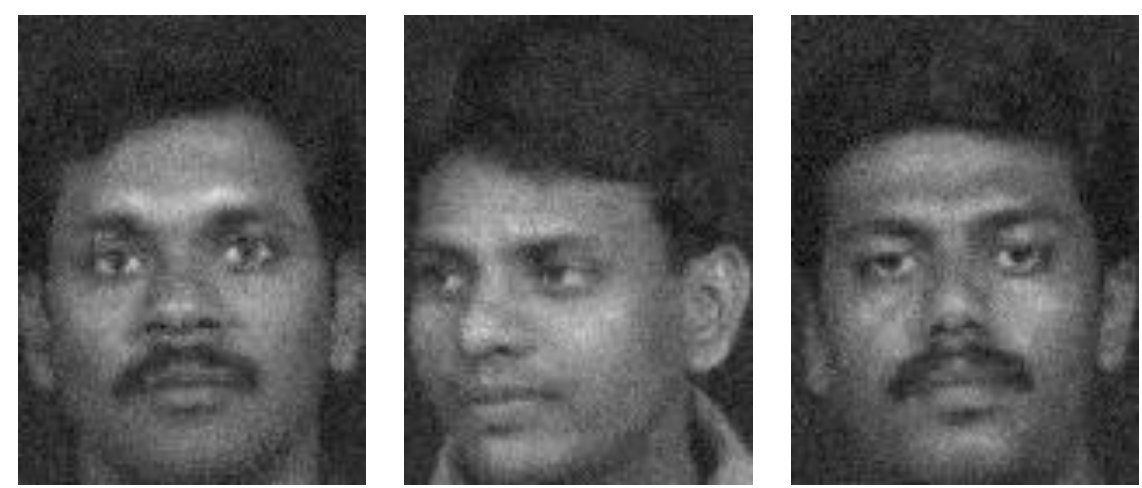

Fig. 7. The pre-processed variants of images dispicted in Figure 6
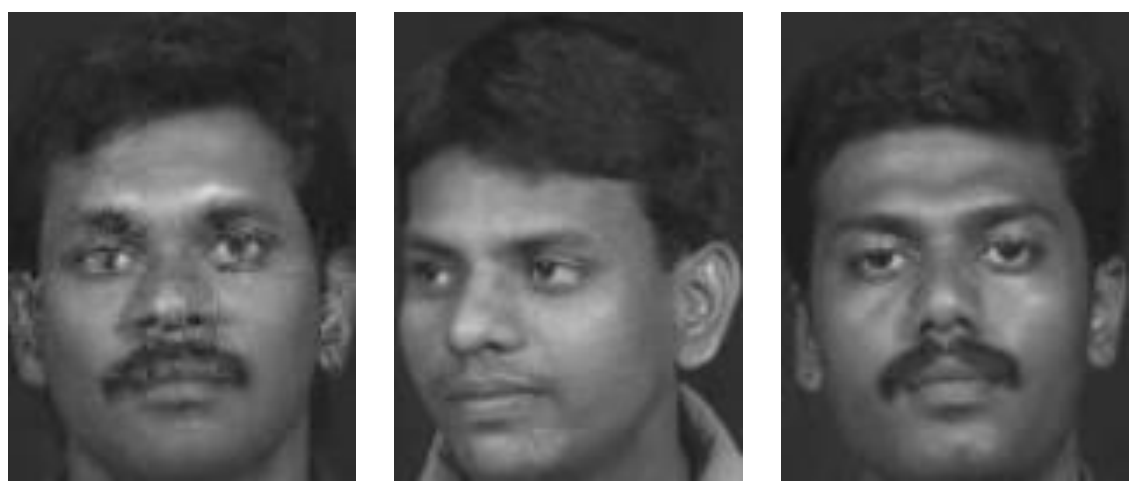

Fig. 8. The resulted cleaned images

Table 2. The qualitative analysis

\begin{tabular}{|l|c|c|}
\hline & SNR-RMS / image & SNR /image \\
\hline $\begin{array}{l}\text { Noisy images ver- } \\
\text { sus original images }\end{array}$ & $8.37,9.03,7.95$ & $18.45,19.11,18.00$ \\
\hline $\begin{array}{l}\text { Cleaned images (us- } \\
\text { ing the pre-processing } \\
\text { step) versus original } \\
\text { images }\end{array}$ & $8.38,11.15,9.59$ & $18.47,20.95,19.63$ \\
\hline $\begin{array}{l}\text { Cleaned images (us- } \\
\text { ing NN's) versus origi- } \\
\text { nal images }\end{array}$ & $12.60,29.27,24.98$ & $22.01,29.32,27.95$ \\
\hline
\end{tabular}




\section{Conclusions and Suggestions for Further Work}

The work reported in the paper aims the development of a new neural network-based methodology for correlated noise removal purposes. The methodology consists in a preprocessing step, where the noise component is partially eliminated, followed by a neural network-based testing/forecasting procedure, applied in order to estimate and remove the still remaining noise. Since during the learning phase the inputs are given by pre-processed compressed images and the outputs represent their corresponding compressed original, cleaned images, each neural network is designed such that, for a given new pre-processed noisy image, to somehow evaluate the quantity of still remaining noise in order to remove it.

The performance of the proposed method is evaluated by a long series of tests, the results being very encouraging, entailing the hope that more sophisticated extensions can be expected to improve it. Some work concerning the use of other compression/decompression methods together with new feature extraction techniques is still in progress.

\section{References}

[1] W. Pratt, Digital Image Processing, $4^{\text {th }}$ Edition, Wiley-Interscience, Hoboden, N.J., 2007

[2] R. Gonzales, R. Woods, Digital Image Processing, $6^{\text {th }}$ Edition, Prentice Hall, 2010

[3] L. State, C. Cocianu, C. Săraru, P. Vlamos, New Approaches in Image Compression and Noise Removal, In: Proceedings of the First International Conference on Advances in Satellite and Space Communications SPACOMM 2009, IEEE Computer Society Press, pp 96-101, 2009

[4] C. Cocianu, L. State and P. Vlamos, On a Certain Class of Algorithms for Noise Removal in Image Processing: A Comparative Study, In: Proceedings of the Third IEEE Conference on Information Technology ITCC-2002, Las Vegas, Nevada, USA, April 8-10, 2002
[5] I.M. Johnstone, B.W. Silverman, Wavelet threshold estimators for data with correlated noise, Journal of the Royal Statistical Society: Series B, Vol. 59, Issue 2, 1997

[6] J. Portilla, V. Strela, M.J. Wainwright, E.P. Simoncelli, Image denoising using scale mixtures of Gaussians in the wavelet domain, IEEE transactions on image processing, Vol. 12, No 11, 1338-51, 2003

[7] B. Goossens, Q. Luong, A. Pizurica, W. Philips, An improved non-local denoising algorithm, In 2008 Proc. Int. Workshop on Local and Non-Local Approximation in Image Processing (LNLA), 1026-3, 2008

[8] N.N. Popomarenko, V.V. Lukin, A.A. Zelensky, J.T. Astola, K.O. Egiazarian, Adaptive DCT-based filtering of images corrupted by spatially correlated noise, Proc. of SPIE-IS\&T Electronic Imaging, SPIE Vol. 6812, 2008

[9] N.N. Popomarenko, V.V. Lukin, K.O. Egiazarian, J.T. Astola, A method for blind estimation of spatially correlated noise characteristics, Proc. SPIE 7532, Image Processing: Algorithms and Systems VIII 753208, 2010

[10] M.P.S. Chawla, PCA and ICA processing methods for removal artifacts and noise in electrocardiograms: A survey and comparision, Applied Soft Computing, 2011

[11] L. Griffanti, G. Salimi-Khorshidi, C.F. Beckmann, E.J. Auerbach, G. Douaud, C.E. Sexton, E. Zsoldos, K.P. Ebmeier, N. Filippini, C.E. Mackay, S. Moeller, J. Xu, E. Yacoub, G. Baselli, K. Ugurbil, K.L. Miller, S.M. Smith, ICA-based artefact removal and accelerated fMRI acquisition for improved Resting State Network imaging, NeuroImage, Elsevier, Vol. 95,2014

[12] P. Hall, J. Jin, Innovated Higher Criticism for Detecting Sparse Signals in Correlated Noise, The Annals of Statistics 2010, Vol. 38, No. 3, pp.1686-1732

[13] G.M. Zhang, D.M. Harvey, D.R. Braden, Signal denoisingn and ultrasonic flaw detection via overcomplete and sparse representation, The Journal of the Acoustical Society of America, 2008 Nov, 124(5) 
[14] S. Abe, Support vector machines for pattern classification, Advances in Pattern Recognition, 2010, pp. 1-473.

[15] C. Cocianu, L. State, P. Vlamos, A New Method for Learning the Support Vector Machines, Proceedings of the $6^{\text {th }}$ International Conference on Software and Data Technology, ICSOFT 2011, INSTICC Press 2011, pp. 365-370

[16] W. Liu, J. Principe and S. Haykin, Kernel Adaptive Filtering: A Comprehensive Introduction, Wiley, 2010.

[17] C. Cocianu, L. State, P. Vlamos, Neural Implementation of a Class of PCA Learning Algorithms, Economic Computation and Economic Cybernetics Studies and Research, No 3,2009
[18] C. Cocianu, A. Stan, Neural Architectures for Correlated Noise Removal in Image Processing, Mathematical Problems in Engineering, Vol. 2016, Hindawi Publishing Corporation

[19] Haykin, S., Neural Networks. A Comprehensive Foundation, Prentice Hall, 2005

[20] K. Ganana, S.N. Deepa, Review on Methods to Fix Number of Hidden Neurons in Neural Networks, Mathematical Problems in Engineering, Vol. 2013, Hindawi Publishing Corporation

[21] http://www.geocities.ws/senthilirtt/Senthil\%20Face $\% 20$ Database\%20Version 1

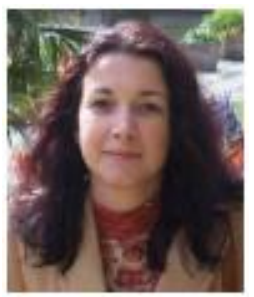

Catalina-Lucia COCIANU, Professor, PhD, currently working with Academy of Economic Studies, Faculty of Cybernetics, Statistics and Informatics, Department of Informatics and Cybernetics in Economy. Competence areas: machine learning, statistical pattern recognition, digital image processing. Research in the fields of pattern recognition, data mining, signal processing. Author of 20 books and more than 100 papers published in national and international journals and conference proceedings.

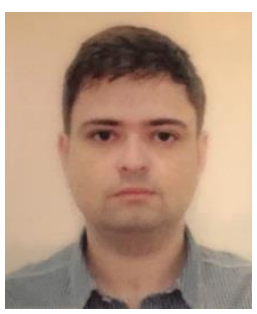

Alexandru Daniel STAN graduated the Faculty of Cybernetics, Statistics and Economic Informatics in 2013. He has graduated the Economic Informatics Master program organized by the Bucharest University of Economic Studies, in 2015. In present he is earning his PhD degree in Economic Informatics with the Doctor's Degree Thesis: "Bio-Inspired Computation in image processing". 